\title{
NEGATIVE VOLATILITY SPILLOVERS IN THE UNRESTRICTED ECCC-GARCH MODEL
}

\author{
ChRISTIAN CONRAD \\ University of Heidelberg \\ Menelaos Karanasos \\ Brunel University
}

\begin{abstract}
This paper considers a formulation of the extended constant or time-varying conditional correlation GARCH model that allows for volatility feedback of either the positive or negative sign. In the previous literature, negative volatility spillovers were ruled out by the assumption that all the parameters of the model are nonnegative, which is a sufficient condition for ensuring the positive definiteness of the conditional covariance matrix. In order to allow for negative feedback, we show that the positive definiteness of the conditional covariance matrix can be guaranteed even if some of the parameters are negative. Thus, we extend the results of Nelson and Cao (1992) and Tsai and Chan (2008) to a multivariate setting. For the bivariate case of order one, we look into the consequences of adopting these less severe restrictions and find that the flexibility of the process is substantially increased. Our results are helpful for the model-builder, who can consider the unrestricted formulation as a tool for testing various economic theories.
\end{abstract}

\section{INTRODUCTION}

The availability of multivariate GARCH models is essential for enhancing our understanding of the relationships between the (co)volatilities of economic and financial time series. For recent surveys on multivariate GARCH specifications and their practical importance in various areas such as asset pricing, portfolio

\footnotetext{
For insightful suggestions and constructive comments we thank Bruce E. Hansen, and two anonymous referees. We would also like to thank Luc Bauwens, Tim Bollerslev, Charles Bos, Michael Burmeister, James Davidson, Russell Davidson, Jurgen A. Doornik, Martin Gassebner, Siem Jan Koopman, Michael J. Lamla, Bent Nielsen, Marius Ooms, Ruey Tsay, and Giovanni Urga for their valuable suggestions. We have also benefited from the comments received from participants at the seminar held in the Department of Economics at the University of Exeter in November 2007, the Inaugural Conference of the Society for Financial Econometrics (New York, 2008), the Annual Conference of the ESRC Econometric Study Group (Bristol, 2008), the $63^{\text {rd }}$ European Meeting of the Econometric Society (Milan, 2008), the Oxford-Man Institute Financial Econometrics and Vast Data Conference (Oxford, 2008), and the $7^{\text {th }}$ OxMetrics User Conference (London, 2008). Address correspondence to Christian Conrad, University of Heidelberg, Faculty of Economics and Social Studies, Bergheimer Str. 58, 69115 Heidelberg, Germany; e-mail: christian.conrad@awi.uni-heidelberg.de. Menelaos Karanasos, Economics and Finance, Brunel University, Uxbridge, West London, UB3 3PH, UK; e-mail: menelaos.karanasos@brunel.ac.uk.
} 
selection, and risk management; see, e.g., Bauwens, Laurent, and Rombouts (2006) and Silvennoinen and Teräsvirta (2007). There are two major problems in specifying a valid multivariate model. First, in many formulations the number of parameters increases quickly with the dimensionality of the model and hence there is a need for parsimonious parameterizations. Second, multivariate specifications have to be parameterized in a way that guarantees the positive definiteness of the conditional covariance matrix almost surely at all points in time. In this article, our focus is on the latter issue. In most of the currently available specifications, positive definiteness is achieved by making rather restrictive assumptions. Either feedback between the conditional variances is completely ruled out, or it is a priori assumed to be positive. For a general class of multivariate GARCH formulations, we derive the necessary and sufficient conditions for the positive definiteness of the covariance matrix and show that these weaker conditions allow for negative volatility feedback.

Bollerslev's (1990) diagonal constant conditional correlation (CCC) GARCH specification is among the most commonly employed multivariate models and serves as a benchmark against which other formulations can be compared. ${ }^{1}$ The diagonal structure implies that each variance behaves as a univariate GARCH process. Hence, the positivity of each conditional variance can be achieved by simply assuming that the parameters of each equation satisfy the conditions derived in Nelson and Cao (1992). However, the main drawback of the diagonal specification is that it rules out potential volatility feedback by assumption. As a consequence, the autocorrelation function of each of the squared observations of the multivariate formulation is no more flexible than that of a univariate GARCH process.

A generalized version of the diagonal CCC model is defined by Jeantheau (1998) and termed extended constant conditional correlation (ECCC)-GARCH by He and Teräsvirta (2004). ${ }^{2}$ In this new formulation, the off-diagonal elements of the matrices are allowed to take positive values (see also Ling and McAleer, 2003). Clearly, under this assumption positive volatility feedback is incorporated into the model. The results of He and Teräsvirta (2004) show that the squared observations of the extended specification have a remarkably richer correlation structure than those of the diagonal one and, hence, are more suited for replicating the manifold features of empirical autocorrelation functions that are observed in practice. The assumption that only positive feedback is allowed for is tempting because positive constants and parameter matrices with nonnegative coefficients are a sufficient condition for the positive definiteness of the conditional covariance matrix in the extended formulation. Since Bollerslev (1986) was the first to impose the nonnegativity restrictions on the parameters of the univariate process, we will refer to the corresponding constraints for the multivariate model as Bollerslev's conditions. He and Teräsvirta (2004) point out that despite the appealing theoretical properties of the extended formulation, more work is needed to find out how useful it is in practice. Subsequently, Nakatani and Teräsvirta (2009) suggest a procedure for testing the hypothesis of a diagonal 
structure against the hypothesis of volatility feedback (of either sign) within the extended framework.

In summary, the currently available specifications are somewhat extreme. That is, at the one extreme, the diagonal model assumes that there is no causal link between the volatilities, whereas, at the other extreme, the extended version (in which the parameters are restricted to being nonnegative) only allows for a positive variance relationship. Leboit, Santa-Clara, and Wolf (2003) point out that "although it can be useful to impose sensible restrictions for forecasting purposes, there is also the danger of employing restrictions that are strongly violated by the data."

At this point, one alternative process suggests itself. That is, we consider a formulation of the extended model that allows for feedback effects between the volatilities, which can be of either sign, positive or negative. We will term this specification the unrestricted ECCC (UECCC)-GARCH. A crucial problem concerns the identification of necessary and sufficient conditions for the unrestricted model to have a positive definite conditional covariance matrix.

Nelson and Cao (1992) derived necessary and sufficient conditions for the positivity of the conditional variance of a univariate $\operatorname{GARCH}(p, q)$ model with $p \leq 2$ and sufficient ones when $p>2$. Recently, Tsai and Chan (2008) have shown that the latter conditions are also necessary. ${ }^{3}$ In this paper we show that the methodologies developed in Nelson and Cao (1992) and Tsai and Chan (2008) can be applied to the $N$-dimensional UECCC-GARCH. We do so by expressing each of the $N$ conditional variances as a "univariate" $\mathrm{ARCH}(\infty)$ specification. That is, each variance admits an infinite moving-average representation in terms of the $N$ convolutions of the GARCH kernels and the corresponding squared errors. Hence, all the $N$ conditional variances are always nonnegative if all the $N^{2}$ kernels are nonnegative. Most importantly, we provide the necessary and sufficient conditions in terms of the parameters of the original process. By checking a finite number of inequality constraints, it can be verified that for a particular set of estimated coefficients, the nonnegativity of all the $N^{2}$ kernels is guaranteed. For practical implementation, we suggest estimating first the unrestricted model and, in case some of the estimated parameters are negative, validating the necessary and sufficient conditions ex-post. ${ }^{4}$ For example, in the bivariate case of order $(1,1)$, we show that at most two parameters of the GARCH matrix can be negative, if and only if they belong to the same column. For this specific case, Nakatani and Teräsvirta (2008) also derive analytical conditions but restrict the two diagonal elements of the GARCH matrix to be positive and, therefore, provide sufficient but not necessary conditions. For the general model, Nakatani and Teräsvirta (2008) suggest to check numerically whether the $N^{2} \mathrm{GARCH}$ kernels in the $\mathrm{ARCH}(\infty)$ representation are nonnegative but do not elaborate on how to obtain the necessary and sufficient conditions analytically.

$\mathrm{He}$ and Teräsvirta (2004) investigate the properties of the auto- and crosscorrelations of the squared errors in the extended model of order $(2,2)$ under the assumption that all the parameters are constrained to be positive. The results in this research suggest that relaxing these constraints allows for more flexibility in 
the shape of the correlation functions. It thus appears that the unrestricted model (the one with possibly negative parameters) may characterize some features of the series that are not adequately captured by the restricted one. ${ }^{5}$

While empirical violations of the Bollerslev constraints might be thought of as resulting either from sampling error or model misspecification, our results show that this is not necessarily the case. Interestingly, they may be in line with economic theory. For example, several theories predict either a positive or a negative association between the variability of inflation and output growth uncertainty (for more details and a review of the literature, see Fountas, Karanasos, and Kim 2006). ${ }^{6}$ Similarly, Caporin (2007) argues that an increase in stock return volatility may lead to a reduction in the variance of volume (see also Karanasos and Kartsaklas, 2009). ${ }^{7}$

The outline of the paper is as follows: Section 2 summarizes some basics concerning the notation used throughout the paper and introduces the unrestricted specification. The main results are stated in Section 3. Section 4 contains an empirical example, and the conclusions can be found in Section 5. Appendix A briefly discusses the second- and fourth-moment structure of the model; Appendix B contains the proofs.

\section{THE MODEL}

\subsection{Notation}

Throughout the paper we will adhere to the following conventions: In order to distinguish matrices (vectors) from scalars, the former are denoted by upper(lower)case boldface symbols. Here $\mathbf{X}(L)=\left[X_{i j}(L)\right]_{i, j=1, \ldots, N}$ denotes an $N \times N$ matrix polynomial in the lag operator $L$, i.e., with $i j$ th element $X_{i j}(L)$ being a polynomial of order $p$. Using standard notation, $\operatorname{det}[\mathbf{X}(L)]$ denotes the determinant, $\mathbf{X}^{j i}(L)$ the $\mathbf{X}(L)$ matrix without its $j$ th row and $i$ th column, and $\operatorname{adj}[\mathbf{X}(L)]=$ $\left[X_{i j}^{\{a\}}(L)\right]_{i, j=1, \ldots, N}$ denotes the adjoint of the $\mathbf{X}(L)$ matrix. That is, $X_{i j}^{\{a\}}(L)=$ $(-1)^{i+j} \operatorname{det}\left[\mathbf{X}^{j i}(L)\right]$ is a scalar polynomial of order $(N-1) \times p$.

Alternatively, we also write the $p$ th order matrix polynomial $\mathbf{X}(L)$ as follows: $\mathbf{X}(L)=\sum_{l=0}^{p} \mathbf{X}^{(l)} L^{l}$, where the upper script with parenthesis denotes an index and the elements of $\mathbf{X}^{(l)}$ are given by the scalars $\left[x_{i j}^{(l)}\right]_{i, j=1, \ldots, N}$.

Furthermore, for square matrices $\mathbf{X}=\left[x_{i j}\right]_{i, j=1, \ldots, N} \in \mathbb{R}^{N \times N}$, we define vec $(\mathbf{X})$ as the $N^{2} \times 1$ vector in which the columns of the square matrix $\mathbf{X}$ are stacked one underneath the other. The symbols $\odot$ and $\otimes$ denote the Hadamard and Kronecker products respectively.

Moreover, $\mathbf{I}_{N}$ denotes the $N \times N$ identity matrix. The transpose and inverse of a matrix are denoted by $\mathbf{X}^{\prime}$ and $\mathbf{X}^{-1}$ respectively. Column vectors will be denoted by lower-case letters, i.e., $\mathbf{x}=\left[x_{i}\right]_{i=1, \ldots, N}$ (unless otherwise indicated) and a diagonal matrix with elements $\left\{x_{1}, \ldots, x_{N}\right\}$ will be denoted by $\operatorname{diag}\{\mathbf{x}\}$. Also, let ${ }^{\wedge}$ and $\mathbb{E}$ denote the elementwise exponentiation and expectation operator, respectively. That is, $\mathbf{X}^{\wedge k}=\left[x_{i j}^{k}\right]_{i, j=1, \ldots, N}$, and $\mathbb{E}(\mathbf{X})=\left[\mathbb{E}\left(x_{i j}\right)\right]_{i, j=1, \ldots, N}$. 
Finally, the matrix (vector) inequality sign $\mathbf{X}>0(\mathbf{x}>0)$ represents elementby-element inequality.

\subsection{Conditional Variances}

In this section we introduce the $\operatorname{UECCC}-\operatorname{GARCH}(p, q)$ model. Consider the $\mathrm{N}$-dimensional weakly stationary vector process

$\mathbf{y}_{t}=\mathbb{E}\left(\mathbf{y}_{t} \mid \mathcal{F}_{t-1}\right)+\boldsymbol{\varepsilon}_{t}$,

where $\mathcal{F}_{t-1}=\sigma\left(\mathbf{y}_{t-1}, \mathbf{y}_{t-2}, \ldots\right)$ is the filtration generated by all the available information through time $t-1$.

We assume that the noise vector $\boldsymbol{\varepsilon}_{t}$ is characterized by the relation

$\boldsymbol{\varepsilon}_{t}=\mathbf{z}_{t} \odot \mathbf{h}_{t}^{\wedge 1 / 2}$

where $\mathbf{h}_{t}=\left[h_{i t}\right]_{i=1, \ldots, N}$ is $\mathcal{F}_{t-1}$ measurable and the stochastic vector $\mathbf{z}_{t}=$ $\left[z_{i t}\right]_{i=1, \ldots, N}$ is independent and identically distributed (i.i.d.) with mean zero and positive definite covariance matrix $\mathbf{R}=\left[\rho_{i j}\right]_{i, j=1, \ldots, N}$ with $\rho_{i j}=1$ for $i=j$. From the above equation, it follows that $\mathbb{E}\left(\boldsymbol{\varepsilon}_{t} \mid \mathcal{F}_{t-1}\right)=\mathbf{0}$ and $\mathbf{H}_{t}=\mathbb{E}\left(\boldsymbol{\varepsilon}_{t} \boldsymbol{\varepsilon}_{t}^{\prime} \mid \mathcal{F}_{t-1}\right)=$ $\operatorname{diag}\left\{\mathbf{h}_{t}^{\wedge 1 / 2}\right\} \mathbf{R} \operatorname{diag}\left\{\mathbf{h}_{t}^{\wedge 1 / 2}\right\}$.

A major problem in specifying a valid multivariate process lies in choosing appropriate parametric specifications for $\mathbf{h}_{t}$ such that $\mathbf{H}_{t}$ is positive definite almost surely for all $t$. Positive definiteness of $\mathbf{H}_{t}$ follows if, in addition to the constant conditional correlation matrix $\mathbf{R}$ being positive definite, the conditional variances $h_{i t}, i=1, \ldots, N$, are positive as well.

Next, we specify the parametric structure of $\mathbf{h}_{t}$. Let $\boldsymbol{\mu}=\left[\mu_{i}\right]_{i=1, \ldots, N}$ be a column vector with finite elements, $\mathbf{B}(L)=\mathbf{I}_{N}-\sum_{l=1}^{p} \mathbf{B}^{(l)} L^{l}$ with $\mathbf{B}^{(l)}=\left[b_{i j}^{(l)}\right]_{i, j=1, \ldots, N}$ and $\mathbf{A}(L)=\sum_{r=1}^{q} \mathbf{A}^{(r)} L^{r}$ with $\mathbf{A}^{(r)}=\left[a_{i j}^{(r)}\right]_{i, j=1, \ldots, N} .^{8}$

We define the vector $\operatorname{GARCH}(p, q)$ process as follows:

$\mathbf{B}(L) \mathbf{h}_{t}=\boldsymbol{\mu}+\mathbf{A}(L) \boldsymbol{\varepsilon}_{t}^{\wedge 2}$.

Obviously, the above process nests Bollerslev's diagonal specification as a special case if we assume that $\mathbf{A}(L)$ and $\mathbf{B}(L)$ are diagonal matrices. Moreover, if it is assumed that all the parameters in expression (3) are positive, then the process corresponds to the ECCC-GARCH model. This assumption guarantees that the conditional variances $h_{i t}, i=1, \ldots, N$, are positive almost surely for all $t$. Although this condition is sufficient for ensuring the positivity of all the conditional variances, the results of Nelson and Cao (1992) and Tsai and Chan (2008) suggest that it is not necessary. In the following, we will investigate whether it is possible to relax this condition by allowing some of the parameters in equation (3) to take negative values while the positivity of all the conditional variances is still ensured. As mentioned in the introduction, the resulting process is termed UECCC-GARCH. 
Remark 1. Although in what follows we will focus our attention on the CCC process, our results hold for the time-varying conditional correlation (TVCC) specification as well. This model differs only in allowing the correlation matrix to be time-varying: $\mathbf{R}_{t}=\left[\rho_{i j, t}\right]_{i, j=1, \ldots, N}$. For examples of such specifications, see Engle (2002), Tse and Tsui (2002), Silvennoinen and Teräsvirta (2009), and Bai and Chen (2008).

Assumption A1 (Identifiability). The formulation of the $N$-dimensional vector $\operatorname{UECCC-GARCH}(p, q)$ model at the true values of the parameters is minimal if $\mathbf{A}(L)$ and $\mathbf{B}(L)$ satisfy the following conditions:

1. $\operatorname{det}[\mathbf{A}(L)] \neq 0$ and $\operatorname{det}[\mathbf{B}(L)] \neq 0$.

2. $\mathbf{A}(L)$ and $\mathbf{B}(L)$ are coprime. That is, any of the greatest common left divisors of $\mathbf{A}(L)$ and $\mathbf{B}(L)$ are unimodular.

3. $\mathbf{A}(L)$ or $\mathbf{B}(L)$ is column reduced. That is, $\operatorname{det}\left[\mathbf{A}^{(q)}\right] \neq 0$ or $\operatorname{det}\left[\mathbf{B}^{(p)}\right] \neq 0$.

Assumption A1 guarantees that the model in equation (3) is identifiable (see Prop. 3.4 in Jeantheau, 1998).

In order to provide a more complete description of the model given by equations (2)-(3) we state in Appendix A: (i) the covariance stationarity assumption, (ii) expressions for the unconditional second moment of the squared errors, and (iii) a condition for the existence of the fourth moment of the bivariate GARCH $(1,1)$ process. However, the results in Appendix A are not needed in the following theoretical development.

\section{NONNEGATIVITY CONSTRAINTS}

We now derive the necessary and sufficient conditions for the positivity of the conditional variances in the $N$-dimensional $\operatorname{UECCC-GARCH}(p, q)$ model. In the first step, we show that each variance admits a "univariate" representation. From this formulation, we obtain an $\mathrm{ARCH}(\infty)$ expansion of each conditional variance in terms of convolutions of GARCH kernels and corresponding squared errors. The nonnegativity of the variances is guaranteed if and only if all the kernels are nonnegative, i.e., if the infinite number of coefficients in the $\mathrm{ARCH}(\infty)$ expansions of the $N^{2}$ kernels are nonnegative. For this, we express these coefficients as functions of the parameters of the original process. It is then shown that checking a finite number of inequality constraints on these parameters ensures the nonnegativity of all GARCH kernels. Special attention is given to the bivariate case, which is relevant for many empirical applications.

\subsection{Univariate Representations}

In order to simplify the description of our analysis, we will introduce the following notation: Set $\beta(L)=1-\sum_{l=1}^{N \times p} \beta_{l} L^{l}=\operatorname{det}[\mathbf{B}(L)]$. Recall, that we have assumed $\beta_{N \times p} \neq 0$, hence $\beta(L)$ is a scalar polynomial of order $N \times p$. Moreover, 
denote by $\phi_{n}, n=1, \ldots, N \times p$, the inverse of the roots of $\beta(z)$. Define $\boldsymbol{\omega}=$ $\left[\omega_{i}\right]_{i=1, \ldots, N}=\operatorname{adj}[\mathbf{B}(L)] \boldsymbol{\mu}$ and $\boldsymbol{\alpha}(L)=\sum_{r=1}^{(N-1) \times p+q} \boldsymbol{\alpha}^{(r)} L^{r}=\operatorname{adj}[\mathbf{B}(L)] \mathbf{A}(L)$ with $\boldsymbol{\alpha}^{(r)}=\left[\alpha_{i j}^{(r)}\right]_{i, j=1, \ldots, N}$, i.e., $\boldsymbol{\alpha}(L)$ is a square matrix polynomial. We can also express it as $\boldsymbol{\alpha}(L)=\left[\alpha_{i j}(L)\right]_{i, j=1, \ldots, N}$ with $\alpha_{i j}(L)=\sum_{r=1}^{(N-1) \times p+q} \alpha_{i j}^{(r)} L^{r}$. Since we have assumed that $\alpha_{i j}^{(N-1) \times p+q} \neq 0$ for all $i, j=1, \ldots, N$, the scalar polynomials $\alpha_{i j}(L)$ are of the order $(N-1) \times p+q$.

Assumption A2 (Invertibility). The inverse roots $\phi_{n}, n=1, \ldots, N \times p$, of $\beta(z)$ lie inside the unit circle and without loss of generality are ordered as follows: $\left|\phi_{1}\right| \geq\left|\phi_{2}\right| \geq \cdots \geq\left|\phi_{N \times p}\right|$.

LEMMA 1. Under Assumptions A1 and A2, the univariate representation of the $N$-dimensional vector $\operatorname{UECCC-GARCH}(p, q)$ process $\mathbf{h}_{t}$ is given by

$\beta(L) \mathbf{h}_{t}=\boldsymbol{\omega}+\boldsymbol{\alpha}(L) \boldsymbol{\varepsilon}_{t}^{\wedge 2}$

Lemma 1 states that we can write each conditional variance $h_{i t}, i=1, \ldots, N$, as being linear in a constant $\omega_{i}$, its own lags $h_{i t-l}, l=1, \ldots, N \times p$, its own lagged squared residuals $\varepsilon_{i t-r}^{2}$ as well as the lagged squared errors $\varepsilon_{j t-r}^{2}$ from the other equations, $j \neq i, r=1, \ldots,(N-1) \times p+q$. Most important, in the univariate representation, $h_{i t}$ no longer depends on lagged values of $h_{j t}$.

Before presenting the general results, we will discuss a specific model in order to make our analysis more concise. Consider the bivariate process of order $(1,1)$ :

$$
\left[\left(\begin{array}{ll}
1 & 0 \\
0 & 1
\end{array}\right)-\left(\begin{array}{ll}
b_{11} & b_{12} \\
b_{21} & b_{22}
\end{array}\right) L\right]\left(\begin{array}{l}
h_{1 t} \\
h_{2 t}
\end{array}\right)=\left(\begin{array}{l}
\mu_{1} \\
\mu_{2}
\end{array}\right)+\left(\begin{array}{ll}
a_{11} & a_{12} \\
a_{21} & a_{22}
\end{array}\right) L\left(\begin{array}{l}
\varepsilon_{1 t}^{2} \\
\varepsilon_{2 t}^{2}
\end{array}\right),
$$

where for typographical convenience we have set $b_{i j}=b_{i j}^{(1)}, a_{i j}=a_{i j}^{(1)}, i, j=1,2$. In matrix form, we have $\left(\mathbf{I}_{2}-\mathbf{B} L\right) \mathbf{h}_{t}=\boldsymbol{\mu}+\mathbf{A} L \boldsymbol{\varepsilon}_{t}^{2}$, with $\mathbf{A}=\left[a_{i j}\right]_{i, j=1,2}$ and $\mathbf{B}=\left[b_{i j}\right]_{i, j=1,2}$.

COROLLARY 1. The univariate representation of the bivariate UECCCGARCH(1,1) process is given by

$$
\begin{aligned}
\left(1-\beta_{1} L-\beta_{2} L^{2}\right)\left(\begin{array}{l}
h_{1 t} \\
h_{2 t}
\end{array}\right)= & \left(\begin{array}{c}
\omega_{1} \\
\omega_{2}
\end{array}\right) \\
+ & {\left[\left(\begin{array}{cc}
\alpha_{11}^{(1)} & \alpha_{12}^{(1)} \\
\alpha_{21}^{(1)} & \alpha_{22}^{(1)}
\end{array}\right) L+\left(\begin{array}{cc}
\alpha_{11}^{(2)} & \alpha_{12}^{(2)} \\
\alpha_{21}^{(2)} & \alpha_{22}^{(2)}
\end{array}\right) L^{2}\right]\left(\begin{array}{c}
\varepsilon_{1 t}^{2} \\
\varepsilon_{2 t}^{2}
\end{array}\right), }
\end{aligned}
$$


with $\beta_{1}=b_{11}+b_{22}, \beta_{2}=b_{12} b_{21}-b_{11} b_{22}$,

$$
\boldsymbol{\omega}=\left(\begin{array}{c}
\left(1-b_{22}\right) \mu_{1}+b_{12} \mu_{2} \\
\left(1-b_{11}\right) \mu_{2}+b_{21} \mu_{1}
\end{array}\right), \quad \boldsymbol{\alpha}^{(1)}=\left(\begin{array}{ll}
a_{11} & a_{12} \\
a_{21} & a_{22}
\end{array}\right)
$$

and $\quad \boldsymbol{\alpha}^{(2)}=\left(\begin{array}{ll}a_{21} b_{12}-a_{11} b_{22} & a_{22} b_{12}-a_{12} b_{22} \\ a_{11} b_{21}-a_{21} b_{11} & a_{12} b_{21}-a_{22} b_{11}\end{array}\right)$.

\subsection{The N-Dimensional Process}

Recall that Jeantheau (1998) assumes that all the coefficients of the $\mathbf{A}^{(r)}$ and $\mathbf{B}^{(l)}$, $(r=1, \ldots, q, l=1, \ldots, p)$ matrices are positive. He and Teräsvirta (2004) point out that a sufficient condition for $\mathbf{h}_{t}>0$ for all $t$ is that all elements in $\boldsymbol{\mu}$ are positive and all elements in the $\mathbf{A}^{(r)}$ and $\mathbf{B}^{(l)}$ matrices are nonnegative for each $r$ and $l$. In addition, by referring to the results of Nelson and Cao (1992), they conjecture that this condition is not necessary, at least not if $p>1$ and/or $q>1$ (see Rmk. 1 in He and Teräsvirta, 2004). By investigating the $\mathrm{ARCH}(\infty)$ representation of the univariate $\operatorname{GARCH}(p, q)$ process, Nelson and Cao (1992) derive necessary and sufficient conditions for the nonnegativity of the conditional variances, which is ensured if and only if all the $\operatorname{ARCH}(\infty)$ coefficients are nonnegative. This, however, does not necessarily mean that all the parameters of the process have to be positive. Next, we derive the $\mathrm{ARCH}(\infty)$ expansion of the vector $\operatorname{UECCC-GARCH}(p, q)$ model.

LEMMA 2. Let Assumptions A1 and A2 be satisfied. Then, equation (4) can be rewritten in the $\mathrm{ARCH}(\infty)$ form:

$\mathbf{h}_{t}=\boldsymbol{\omega} / \beta(1)+\Psi(L) \boldsymbol{\varepsilon}_{t}^{\wedge 2}$,

where $\Psi(L)=\left[\Psi_{i j}(L)\right]_{i, j=1, \ldots, N}=\boldsymbol{\alpha}(L) / \beta(L)$ with $\Psi_{i j}(L)=\sum_{k=1}^{\infty} \psi_{i j}^{(k)} L^{k}=$ $\alpha_{i j}(L) / \beta(L)$.

Here, each $\Psi_{i j}(L)$ can be thought of as an $\mathrm{ARCH}(\infty)$ kernel of a GARCH model of the order $(N \times p,(N-1) \times p+q)$.

For illustrative purposes, consider again the bivariate process of order $(1,1)$. If Assumptions A1 and A2 hold, then from equation (6) it follows that the $\mathrm{ARCH}(\infty)$ representation of the process exists and is given by

$$
\left(\begin{array}{l}
h_{1 t} \\
h_{2 t}
\end{array}\right)=\left(\begin{array}{c}
\omega_{1} /\left(1-\beta_{1}-\beta_{2}\right) \\
\omega_{2} /\left(1-\beta_{1}-\beta_{2}\right)
\end{array}\right)+\left(\begin{array}{ll}
\Psi_{11}(L) & \Psi_{12}(L) \\
\Psi_{21}(L) & \Psi_{22}(L)
\end{array}\right)\left(\begin{array}{c}
\varepsilon_{1 t}^{2} \\
\varepsilon_{2 t}^{2}
\end{array}\right),
$$


where each of the four kernels $\Psi_{i j}(L)$ corresponds to an $\mathrm{ARCH}(\infty)$ kernel of a univariate $\operatorname{GARCH}(2,2)$ process:

$\Psi_{i j}(L)=\frac{\alpha_{i j}^{(1)} L+\alpha_{i j}^{(2)} L^{2}}{\left(1-\beta_{1} L-\beta_{2} L^{2}\right)}=\sum_{k=1}^{\infty} \psi_{i j}^{(k)} L^{k} \quad$ for $i, j=1,2$.

Following the proof of Proposition 1 in Conrad and Haag (2006), we can recursively express each $\psi_{i j}^{(k)}$ sequence as $\psi_{i j}^{(k)}=\beta_{1} \psi_{i j}^{(k-1)}+\beta_{2} \psi_{i j}^{(k-2)}$ for $k \geq 3$, where $\psi_{i j}^{(1)}=\alpha_{i j}^{(1)}$ and $\psi_{i j}^{(2)}=\beta_{1} \alpha_{i j}^{(1)}+\alpha_{i j}^{(2)}$. Obviously, the $\psi_{i j}^{(k)}$, s can now be expressed in terms of the $a$ 's and $b$ 's using Corollary 1. For example, $\psi_{11}^{(1)}=a_{11}$ and $\psi_{11}^{(2)}=a_{11} b_{11}+a_{21} b_{12}$.

Clearly, for the $N$-dimensional process in equation (3) to be well-defined and the $N$ conditional variances to be positive almost surely for all $t$, all the constants $\omega_{i}$ must be positive and all the $\psi_{i j}^{(k)}$ coefficients in the $\mathrm{ARCH}(\infty)$ representation, that is equation (7), must be nonnegative: $\psi_{i j}^{(k)} \geq 0, i, j=1, \ldots, N$, for $k=1,2, \ldots$.

In practice, given a particular set of parameters, checking the nonnegativity of $\left\{\psi_{i j}^{(k)}\right\}_{k=1}^{\infty}, i, j=1, \ldots, N$, may be a numerically infeasible task. In the following theorem we show that under some conditions, the nonnegativity of $\left\{\psi_{i j}^{(k)}\right\}_{k=1}^{k_{i j}^{*}}$ for some tractable integers $k_{i j}^{*}$ is necessary and sufficient for the nonnegativity of $\left\{\psi_{i j}^{(k)}\right\}_{k=1}^{\infty}$.

THEOREM 1. Consider the $N$-dimensional vector $\operatorname{UECCC-GARCH(p,q)}$ model in equation (3) and let Assumptions A1 and A2 be satisfied and all the inverse roots $\phi_{n}, n=1, \ldots, N \times p$, be distinct. Then the following conditions are necessary and sufficient for $h_{i t}>0, i=1, \ldots, N$, for all $t$ :

(a) $\omega_{i}>0$ for all $i=1, \ldots, N$.

(b) $\phi_{1}$ is real, and $\phi_{1}>0$,

$$
\begin{aligned}
\alpha_{i j}\left(\phi_{1}^{-1}\right) & >0, \quad \text { for } i, j=1, \ldots, N, \\
\psi_{i j}^{(k)} & \geq 0, \quad \text { for } i, j=1, \ldots, N \text { and } k=1, \ldots, k_{i j}^{*},
\end{aligned}
$$

where $k_{i j}^{*}$ is the smallest integer greater than or equal to max $\{0, \varphi\}$ with

$$
\begin{aligned}
\varphi & \left.=\left\{\log \left(\eta_{i j}^{(1)}\right)-\log (N p-1) \eta_{i j}^{*}\right)\right\} /\left\{\log \left(\left|\phi_{2}\right|\right)-\log \left(\phi_{1}\right)\right\}, \\
\eta_{i j}^{*} & =\max _{2 \leq n \leq N \times p}\left|\eta_{i j}^{(n)}\right|, \quad \text { and } \quad \eta_{i j}^{(n)}=-\frac{\alpha_{i j}\left(\phi_{n}^{-1}\right)}{\beta^{\prime}\left(\phi_{n}^{-1}\right)}, \quad 1 \leq n \leq N \times p,
\end{aligned}
$$

where $\beta^{\prime}(z)$ denotes the first derivative of $\beta(z)$.

The proof of Theorem 1 relies on the observation that the results of Tsai and Chan (2008) can be applied separately to each of the $N^{2}$ GARCH kernels $\Psi_{i j}(L)$. 
Hence, we reduce an infinite number of inequality constraints on the $\operatorname{ARCH}(\infty)$ coefficients to a finite number of conditions on the parameters of the process.

For the general model, Nakatani and Teräsvirta (2008, Thm. 2) also argue that all the $\left\{\psi_{i j}^{(k)}\right\}_{k=1}^{\infty}$ coefficients have to be nonnegative. However, their statement is not 'constructive' in the sense of our Theorem 1, because they were not able to reduce the infinite number of inequality constraints on the $\psi_{i j}^{(k)}$ to a finite number of constraints on the parameters of the underlying process.

As mentioned in Remark 1, the results for the UECCC model hold also for any parametrization of the unrestricted extended TVCC formulation. In addition, it is straightforward to see that they hold for the asymmetric power version of the UECCC as well. ${ }^{9}$

\subsection{The Bivariate Process of Order $(1,1)$}

Because the bivariate model of order $(1,1)$ is definitely the most often applied specification, we intensively discuss the corresponding inequalities and their interpretation.

PROPOSITION 1. Let Assumptions A1 and A2 be satisfied and $\phi_{1} \neq \phi_{2}$. The following conditions are necessary and sufficient for $h_{i t}>0, i=1,2$, for all $t$ in the bivariate UECCC-GARCH(1,1) model:

(a) For the two constants, we require

$$
\omega_{1}=\left(1-b_{22}\right) \mu_{1}+b_{12} \mu_{2}>0 \quad \text { and } \quad \omega_{2}=\left(1-b_{11}\right) \mu_{2}+b_{21} \mu_{1}>0 .
$$

(b) Condition (C1) in Theorem 1 reduces to

$$
\left(b_{11}-b_{22}\right)^{2}>-4 b_{12} b_{21} \text { and } \phi_{1}>0 .
$$

Condition (C2) becomes

$$
\begin{aligned}
& \alpha_{11}\left(\phi_{1}^{-1}\right)=\left(b_{11}-\phi_{2}\right) a_{11}+b_{12} a_{21}>0, \\
& \alpha_{12}\left(\phi_{1}^{-1}\right)=\left(b_{11}-\phi_{2}\right) a_{12}+b_{12} a_{22}>0, \\
& \alpha_{21}\left(\phi_{1}^{-1}\right)=b_{21} a_{11}+\left(b_{22}-\phi_{2}\right) a_{21}>0, \\
& \alpha_{22}\left(\phi_{1}^{-1}\right)=b_{21} a_{12}+\left(b_{22}-\phi_{2}\right) a_{22}>0 .
\end{aligned}
$$

Since $k_{i j}^{*}=2$, Condition (C3) amounts to

$$
\begin{array}{ll}
\psi_{11}^{(1)}=a_{11} \geq 0, & \psi_{12}^{(1)}=a_{12} \geq 0, \\
\psi_{21}^{(1)}=a_{21} \geq 0, & \psi_{22}^{(1)}=a_{22} \geq 0,
\end{array}
$$

and

$$
\begin{array}{ll}
\psi_{11}^{(2)}=b_{11} a_{11}+b_{12} a_{21} \geq 0, & \psi_{12}^{(2)}=b_{11} a_{12}+b_{12} a_{22} \geq 0, \\
\psi_{21}^{(2)}=b_{21} a_{11}+b_{22} a_{21} \geq 0, & \psi_{22}^{(2)}=b_{21} a_{12}+b_{22} a_{22} \geq 0 .
\end{array}
$$


Note that if $\phi_{2}>0$, then $\alpha_{i j}\left(\phi_{1}^{-1}\right)>0$, that is condition $\left(C 2^{\prime}\right)$, directly implies $\psi_{i j}^{(2)} \geq 0$, that is condition $\left(C 3^{\prime} b\right)$, and vice versa if $\phi_{2}<0$.

In the bivariate model, both conditional variances are always positive if the two constants $\omega_{1}$ and $\omega_{2}$ are positive and all four $\operatorname{GARCH}(2,2)$ kernels are nonnegative. Note that the conditions that Proposition 1 places on each of the four kernels are equivalent to the ones derived in Nelson and Cao (1992) and He and Teräsvirta (1999) (see their Assump. (A23)) for the univariate GARCH(2,2) model. However, the four kernels are functions of the same underlying parameters of the bivariate process, and the conditions on the four kernels have to be satisfied simultaneously. It is clear that the conditions of Proposition 1 are satisfied if all parameters are assumed to be positive. Finally, it should be noted that conditions $\left(\mathrm{C}^{\prime} \mathrm{a}\right)$ and $\left(\mathrm{C}^{\prime}\right)-\left(\mathrm{C}^{\prime} \mathrm{b}\right)$ can be written compactly in a matrix form as $\mathbf{A} \geq 0$ and $\left[\mathbf{B}-\max \left(\phi_{2}, 0\right) \mathbf{I}_{2}\right] \mathbf{A}>0$, respectively.

Nelson and Cao (1992) have shown that under the necessary and sufficient conditions for the univariate $\operatorname{GARCH}(2,2)$ process with parameters $\omega, \alpha_{1}, \alpha_{2}$, $\beta_{1}$, and $\beta_{2}$, at most two out of the five parameters may be negative. These are the second-order coefficients, i.e., $\alpha_{2}$ and $\beta_{2}$. With the following corollaries, we will investigate which restrictions Proposition 1 imposes on the parameters of the bivariate model.

First, note from Condition ( $\mathrm{C}^{\prime} \mathrm{a}$ ) that all four elements in A must be nonnegative. This is not surprising, since the coefficients of $\mathbf{A}$ are the first-order ARCH parameters, $\alpha_{i j}^{(1)}$, of the $\operatorname{GARCH}(2,2)$ kernels in the univariate representations (see Corollary 1) and, as proved in Nelson and Cao (1992), those should be nonnegative. Actually, this holds for the $N$-dimensional process of order $(p, q)$ as well (see also Nakatani and Teräsvirta, 2008). Hence, only coefficients in $\boldsymbol{\mu}$ and $\mathbf{B}$ may be allowed to be negative.

COROLLARY 2. In the bivariate UECCC-GARCH(1,1) model, both diagonal elements of $\mathbf{B}$ cannot be negative simultaneously.

Again, Corollary 2 does not come as a surprise, because $\beta_{1}=b_{11}+b_{22}$ is the first-order GARCH parameter of the four kernels in the univariate representations and according to Nelson and Cao (1992), $\beta_{1}$ has to be nonnegative.

It is worth noting that while a negative value of, say, $b_{11}$ might suggest that the higher $h_{1 t-1}$, the lower $h_{1 t}$, this is only a partial effect. The univariate representation implies $\beta_{1}=b_{11}+b_{22}>0$ and hence an "overall" positive relation between $h_{1 t-1}$ and $h_{1 t}$.

COROLLARY 3. In the bivariate UECCC-GARCH $(1,1)$ process, both elements of one row of $\mathbf{B}$ cannot be negative simultaneously.

COROLLARY 4. In the bivariate UECCC-GARCH(1,1) specification, negative volatility feedback in both directions $\left(b_{12}<0\right.$ and $\left.b_{21}<0\right)$ is ruled out. 
Corollary 4 states an important result, because it shows the model's limitations: Economic theories that imply negative volatility spillovers in both equations cannot be tested within the bivariate framework. ${ }^{10}$

\section{Example 1}

Tsay (2002, p. 369) applied the model in equation (5) to monthly returns of IBM stock and the S\&P 500 index from January 1926 to December 1999. He obtained the following estimates for the entries of the $\mathbf{B}$ matrix: $b_{11}=0.873, b_{12}=-0.031$, $b_{21}=-0.066$, and $b_{22}=0.913$. Since both off-diagonal elements are negative, the estimated model does not guarantee the positive definiteness of $\mathbf{H}_{t}$.

Suppose $b_{12}$ and $b_{21}$ were negative, while $b_{11}$ and $b_{22}$ as well as the elements in A were positive. Such a constellation would imply that all second-order ARCH coefficients in the four $\operatorname{GARCH}(2,2)$ kernels are negative; that is, $\boldsymbol{\alpha}^{(2)}<0$ in Corollary 1 , but it is ruled out by Corollary 4 . However, the case that all four second-order ARCH coefficients are negative simultaneously is not ruled out in general, because it can arise even if all parameters are positive.

COROLLARY 5. In the bivariate UECCC-GARCH(1,1) model, at most two elements of $\mathbf{B}$ can be negative, if and only if they belong to the same column.

More specifically, if either $b_{i j}<0$ or $b_{j j}<0$ or both, $i, j=1,2, i \neq j$, then under $\left(\mathrm{C}^{\prime}\right)$ and $\left(\mathrm{C}^{\prime} \mathrm{a}\right)$, conditions $\left(\mathrm{C}^{\prime}\right)$ and $\left(\mathrm{C}^{\prime} \mathrm{b}\right)$ reduce to

$b_{i i} \geq 0, \frac{b_{j i}}{\left|b_{j j}-\max \left(\phi_{2}, 0\right)\right|} \geq \max _{(l=1,2)}\left(\frac{a_{j l}}{a_{i l}}\right), \quad i, j=1,2, \quad i \neq j$.

The result in equation (10) covers the case where one of the off-diagonal elements of $\mathbf{B}$ is negative while all other parameters are positive. This case is particularly interesting because it implies that $\beta_{2}=b_{12} b_{21}-b_{11} b_{22}<0$ and both second-order ARCH coefficients in one row of $\boldsymbol{\alpha}^{(2)}$ are negative. E.g., if $b_{12}<0$, this implies that $\alpha_{11}^{(2)}$ and $\alpha_{12}^{(2)}$ in equation (6) are negative. This special case is examined by Nakatani and Teräsvirta (2008, Cor. 4), who restrict the diagonal GARCH parameters to be positive.

\section{Example 2}

Bai and Chen (2008) apply the bivariate unrestricted extended TVCC-GARCH $(1,1)$ model to the data set used in Tsay (2002, p. 374). They use the following Cholesky decomposition: $\mathbf{H}_{t}=\mathbf{L}_{t} \mathbf{G}_{t} \mathbf{L}_{t}^{\prime}$, where $\mathbf{L}_{t}=\left(\begin{array}{cc}1 & 0 \\ q_{t} & 1\end{array}\right), \mathbf{G}_{t}=\left(\begin{array}{cc}h_{1 t} & 0 \\ 0 & g_{2 t}\end{array}\right)$, with $q_{t}=\rho_{12, t} \sqrt{h_{2 t}} / \sqrt{h_{1 t}}$ and $g_{2 t}=h_{2 t}\left(1-\rho_{12, t}^{2}\right)$. Then the bivariate process: $\left[\mathbf{I}_{2}-\mathbf{B} L\right] \mathbf{g}_{t}=\boldsymbol{\mu}+\mathbf{A} \boldsymbol{\eta}_{t-1}^{\wedge 2}$, where $\mathbf{g}_{t}=\left(\begin{array}{ll}h_{1 t} & g_{2 t}\end{array}\right)^{\prime}, \boldsymbol{\eta}_{t}^{\prime}=\left(\begin{array}{ll}\varepsilon_{1 t} & \eta_{2 t}\end{array}\right)$ with $\eta_{2 t}=$ $\varepsilon_{2 t}-q_{t} \varepsilon_{1 t}$ is estimated. ${ }^{11}$ The following estimates for the entries of the $\mathbf{A}$ and B matrices are obtained: $\mathbf{A}=\left(\begin{array}{ll}0.113 & - \\ 0.021 & 0.052\end{array}\right), \mathbf{B}=\left(\begin{array}{cc}0.804 & - \\ -0.040 & 0.937\end{array}\right)$. Since $b_{12}=0$, we have $\phi_{1}=b_{22}=0.937$ and $\phi_{2}=b_{11}=0.804>0$. Most important, 
condition $\left(\mathrm{C}^{\prime}\right)$ is violated because $b_{21} a_{11}+\left(b_{22}-\phi_{2}\right) a_{21}=-0.115<0$. Therefore, the estimated model does not guarantee the positive definiteness of either $\mathbf{G}_{t}$ or $\mathbf{H}_{t}$.

\section{Example 3}

Nakatani and Teräsvirta (2008) assume that the conditional variances of two Japanese stock return series can be characterized by a bivariate ECCC-GARCH $(1,1)$ process and obtain the following result:

$\mathbf{h}_{t}=\left(\begin{array}{l}0.1288 \\ 0.0541\end{array}\right)+\left(\begin{array}{ll}0.1018 & 0.0350 \\ 0.0341 & 0.0394\end{array}\right) \boldsymbol{\varepsilon}_{t-1}^{\wedge 2}+\left(\begin{array}{cc}0.8093 & 0.0353 \\ -0.0467 & 0.9627\end{array}\right) \mathbf{h}_{t-1}$,

with $\rho_{12}=0.6109$. Most importantly, the estimated value of the parameter $b_{21}$ is negative and highly significant (standard errors can be found in Nakatani and Teräsvirta, 2008). Clearly, this parameter combination satisfies the conditions of Proposition 1 and highlights the empirical relevance of negative volatility feedback.

To summarize our results concerning the elements of $\mathbf{B}$ figuratively, we may say that at most two out of the four coefficients of the $\mathbf{B}$ matrix can be negative as long as they are elements of the same column.

Further, unlike the univariate $\operatorname{GARCH}(2,2)$ model, the bivariate $\operatorname{GARCH}(1,1)$ can produce, under some conditions, negative values for the constants. The case that one of the two is negative is straightforward and requires no further discussion. We now consider the case of both constants being negative. First note that the condition $\phi_{i}<1$ for $i=1,2$ implies that $b_{11}$ and $b_{22}$ cannot be greater than one simultaneously, because $b_{11}+b_{22}=\phi_{1}+\phi_{2}$.

COROLLARY 6. In the bivariate UECCC-GARCH(1,1) model, the constants $\mu_{1}$ and $\mu_{2}$ can be negative simultaneously if and only if one of the two diagonal elements of $\mathbf{B}$ is greater than one.

Finally, we should emphasize that the set of conditions provided by the above Proposition 1 is weaker than those in Jeantheau (1998), He and Teräsvirta (2004), and Nakatani and Teräsvirta $(2008,2009)$. That is, although all four ARCH coefficients must be nonnegative, the two constants and two out of the four GARCH parameters, under some conditions, can be negative.

These results require more discussion. For illustrative purposes in the next subsection we examine a few numerical examples.

\subsection{Numerical Examples}

In what follows we graphically illustrate the necessary and sufficient parameter set for the bivariate UECCC-GARCH $(1,1)$ model. This will provide a better understanding of the results presented in the previous subsection. We discuss four examples. 
In the first example, we allow the two off-diagonal elements of $\mathbf{B}$ to vary. The parameters chosen for Example 1 are rather standard, except that we assume $a_{21}=0.20$, which implies that the squared innovations $\varepsilon_{1 t-1}^{2}$ have a strong impact on $h_{2 t}$. In Example 2 we examine the situation where $b_{11}$ and $b_{21}$ (i.e., two coefficients in the first column of $\mathbf{B}$ ) vary. In Example 3 the two constants $\mu_{1}$ and $\mu_{2}$ vary freely, while the two off-diagonal elements of $\mathbf{A}$ vary in Example 4.

In the following figures, the bold solid lines show which combinations of the two freely varying parameters satisfy the necessary and sufficient conditions of Proposition 1 when the other parameters are fixed as in the examples of Table 1. Additionally, they show which combinations satisfy the conditions for the existence of the unconditional second (dotted lines) and fourth moments (dashed lines), which are discussed in Appendix A.

We begin by discussing the implications of Example 1, which is presented in Figure 1a. First, all combinations of $b_{12}$ and $b_{21}$ that are bounded by the three bold solid lines satisfy the conditions of Proposition 1. The line in quadrant one represents the invertibility Assumption A2, which is satisfied when $b_{12}<$ $\left(1-b_{11}\right)\left(1-b_{22}\right) / b_{21}=0.14 / b_{21}$. In quadrant four, the line stands for the condition that $\phi_{1}$ is real: $b_{12}<-\left(b_{11}-b_{22}\right)^{2} /\left(4 b_{21}\right)=-0.062 / b_{21}$. The requirement that $b_{12}$ is nonnegative is triggered by condition $\left(\mathrm{C} 2^{\prime}\right): b_{12}>a_{11}\left|b_{11}-\phi_{2}\right| / a_{21}=$ $\left|0.30-\phi_{2}\right| / 6.67$. In line with Corollary 4 , this ensures that not both off-diagonal elements of $\mathbf{B}$ can be negative simultaneously. Finally, it is evident that the Bollerslev conditions that would restrict both $b_{12}$ and $b_{21}$ to being positive are too strong, since they exclude the area below the bold solid line in quadrant four where $b_{21}<0$. Second, the combinations of $b_{12}$ and $b_{21}$, which are bounded by the dotted lines, satisfy the conditions for the existence of the second moments. The dotted lines in quadrant one and four represent the covariance stationarity condition (see Assumption A3 in Appendix A), while the straight dotted line in quadrants two and three is triggered by the restriction that the unconditional variances are nonnegative (see equation (A.2) in Appendix A). Similarly, the constraints for the existence of the fourth moments imply the dashed lines (see equations (A.3) and (A.4) in Appendix A). In the first quadrant, the restrictions implied by the conditions for the existence of the second and fourth moments are more

TABLE 1. Data generating processes (DGP) for Examples 1 to 4

\begin{tabular}{cccccc}
\hline & DGP Ex. 1 & DGP Ex. 2 & DGP Ex. 3 & DGP Ex. 4 \\
\hline $\boldsymbol{\mu}^{\prime}$ & $\left(\begin{array}{ll}0.10 & 0.20\end{array}\right)$ & $\left(\begin{array}{ll}0.10 & 0.20\end{array}\right)$ & $\left(\begin{array}{ll}\mu_{1} & \mu_{2}\end{array}\right)$ & $\left(\begin{array}{ll}0.20 & 0.10\end{array}\right)$ \\
$\mathbf{A}$ & $\left(\begin{array}{ll}0.03 & 0.02 \\
0.20 & 0.05\end{array}\right)$ & $\left(\begin{array}{ll}0.03 & 0.02 \\
0.40 & 0.05\end{array}\right)$ & $\left(\begin{array}{cc}0.07 & 0.03 \\
0.01 & 0.002\end{array}\right)$ & $\left(\begin{array}{cc}0.03 & a_{12} \\
a_{21} & 0.05\end{array}\right)$ \\
$\mathbf{B}$ & $\left(\begin{array}{ll}0.30 & b_{12} \\
b_{21} & 0.80\end{array}\right)$ & $\left(\begin{array}{ll}b_{11} & 0.30 \\
b_{21} & 0.80\end{array}\right)$ & $\left(\begin{array}{cc}1.20 & -0.50 \\
0.50 & 0.15\end{array}\right)$ & $\left(\begin{array}{cc}0.10 & 0.30 \\
-0.35 & 0.80\end{array}\right)$ \\
\hline
\end{tabular}



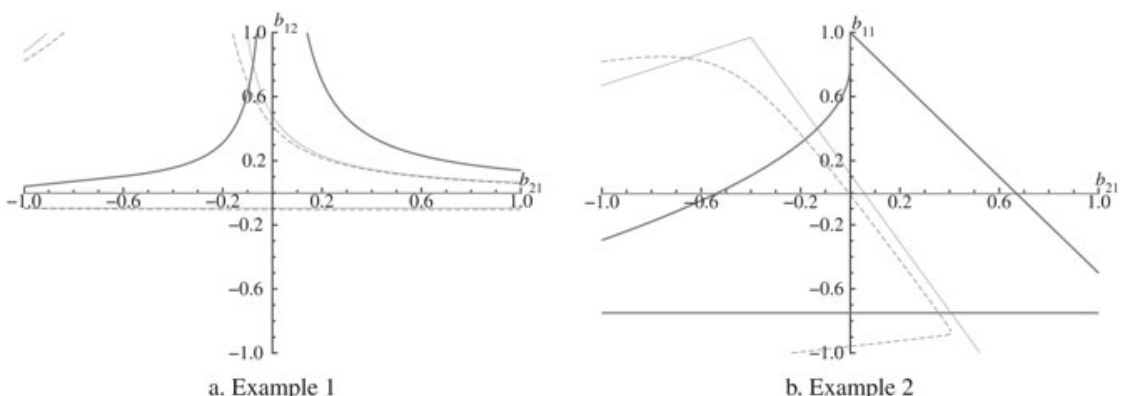

a. Example 1

b. Example 2

FIGURE 1. Necessary and sufficient parameter sets for bivariate UECCC-GARCH $(1,1)$ from Examples 1 (panel a) and 2 (panel b). Bold solid lines represent the restrictions implied by Proposition 1; dotted and dashed lines stand for the restrictions for the existence of the unconditional second and fourth moments.

restrictive than the one implied by the invertibility Assumption A2. In summary, the parameter set that satisfies all conditions simultaneously is given by the area that is below the dashed line in the first and fourth quadrant and below the bold solid line in the fourth quadrant.

Next, we consider the case where both elements of the first column of B vary. The bold solid lines in Figure 1b show the necessary and sufficient set for Example 2. As suggested by Corollary 5, we can now have both elements in a column being negative. The line in quadrants one and two represents the invertibility Assumption A2: $b_{11}<1-b_{12} b_{21} /\left(1-b_{22}\right)=1-1.50 b_{21}$; while the one in quadrants two and three is triggered by condition $\left(\mathrm{C}^{\prime} \mathrm{b}\right): b_{11} \geq-b_{12} a_{22} / a_{12}=-0.75$. The line in quadrant four represents condition $\left(\mathrm{C}^{\prime}\right): b_{21}>-\left(b_{22}-\phi_{2}\right) a_{22} / a_{12}=$ $-2.50\left(0.80-\phi_{2}\right)$.

Figure 2a shows that, for the parameters in Example 3, both constants can be negative. All parameter combinations above the bold solid lines in quadrants one and three are valid: $\mu_{1}>\left|b_{12}\right| \mu_{2} /\left(1-b_{22}\right)=0.59 \mu_{2}$ and $\mu_{1}>\left|1-b_{11}\right| \mu_{2} / b_{21}=$ $0.40 \mu_{2}$, respectively. Interestingly, combinations with $\mu_{1}<0, \mu_{2}>0$ are ruled out, whereas, in sharp contrast, all possible combinations with $\mu_{1}>0, \mu_{2}<0$ are permitted. Example 3 is in line with Corollary 6, which requires, for the case $\mu_{1}<0, \mu_{2}<0$, that one of the diagonal elements of $\mathbf{B}$ must be greater than one $\left(b_{11}=1.2\right)$. Example 4 is visualized in Figure $2 \mathrm{~b}$. The conditions of Proposition 1 allow for negative volatility feedback from $h_{1 t}$ to $h_{2 t}$ only if $a_{12}$ takes rather small values. In particular, since $\phi_{2}=0.32>0$, the two off-diagonal elements of $\mathbf{A}$ should satisfy condition $\left(\mathrm{C}^{\prime}\right): a_{21}>a_{11}\left|b_{11}-\phi_{2}\right| / b_{12}=0.02$ and $a_{12}<$ $a_{22} b_{12} /\left|b_{11}-\phi_{2}\right|=0.07$.

The examples above impressively show that the weaker conditions of Proposition 1 substantially enlarge the necessary and sufficient parameter set in comparison to the Bollerslev conditions. Having a wider admissible parameter set should increase the flexibility of the UECCC-GARCH $(1,1)$ model. Assuming conditional normality and $\rho_{12}=0.2$, we illustrate this for Example 2 by plotting 


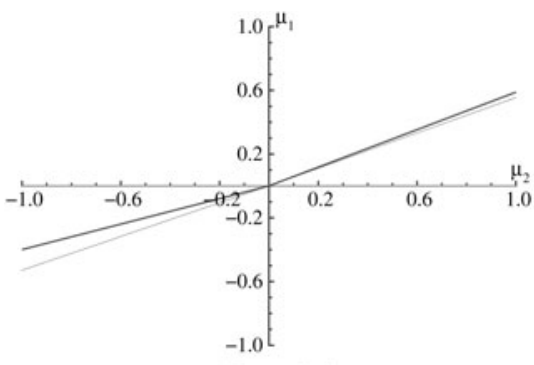

a. Example 3

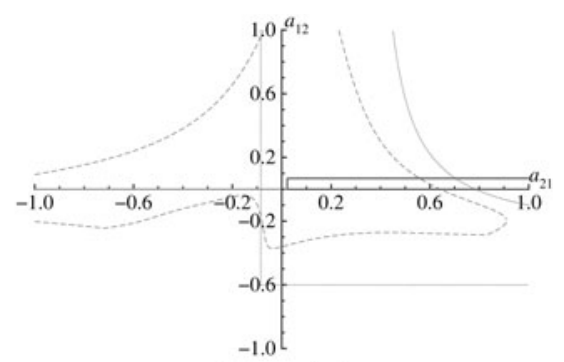

b. Example 4

FIGURE 2. Necessary and sufficient parameter sets for bivariate UECCC-GARCH $(1,1)$ from Examples 3 (panel a) and 4 (panel b). The bold solid lines represent the restrictions implied by Proposition 1 . The dotted and dashed lines stand for the restrictions for the existence of the unconditional second and fourth moments.

the auto- and cross-correlations of the squared errors. ${ }^{12}$ For this, we set $b_{21}=$ -0.10 and vary $b_{11}$ from -0.70 to 0 by steps of 0.10 . Since $b_{22}=0.80$, the restriction that $\beta_{1}=b_{11}+b_{22}>0$ is always satisfied. When $b_{11}$ takes large negative values, we observe an oscillating behavior in the auto- and cross-correlations $r_{11}(m)$ and $r_{12}(m), m=1,2, \ldots$, which disappears as $b_{11}$ gets close to zero.

\section{TESTING ECONOMIC THEORY}

The debate about the inflation-growth interaction is linked to another ongoing dispute, that of the existence or absence of a variance relationship. As Fuhrer (1997) puts it: "It is difficult to imagine a policy that embraces targets for the level of inflation or output growth without caring about their variability around their target levels. The more concerned the monetary policy is about maintaining the level of an objective at its target, the more it will care about the variability of that objective around its target."

There are many controversies in the theoretical literature on the relationship between the four variables (see Fountas, Karanasos, and Kim, 2006, and the references therein). The extent to which there is an interaction of either sign between the two variances is an issue that cannot be resolved on merely theoretical grounds. Furthermore, the models regarding the "uncertainty link" that do exist are often ambiguous in their predictions. These considerations reinforce a widespread awareness of the need for more empirical evidence, but they also make clear that a good empirical framework is lacking. In what follows, we will employ the bivariate process to examine how U.S. nominal and real uncertainties are interrelated.

Monthly U.S. data for the period 1960:01 to 2007:11 are obtained from Datastream to provide a reasonable number of observations. The inflation rate $\left(y_{1 t}\right)$ and output growth $\left(y_{2 t}\right)$ series are calculated as the monthly difference in the 

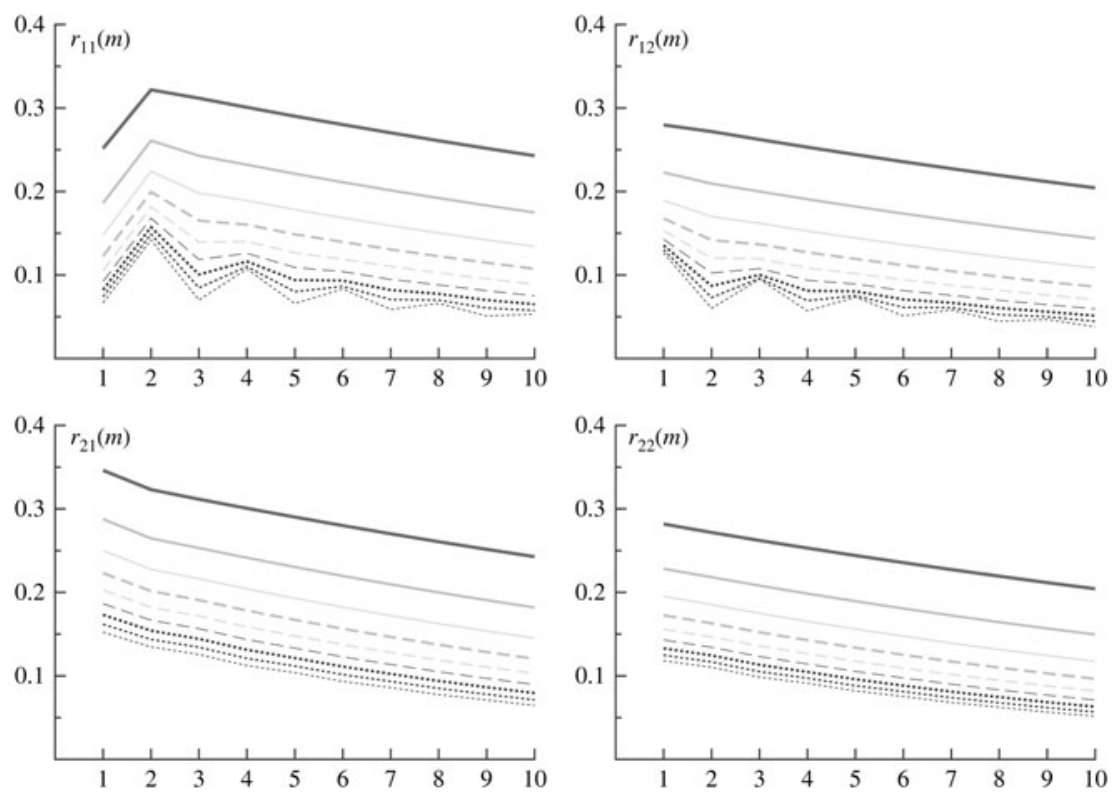

FigURE 3. The figure shows the $m$ th order auto- and cross-correlations, $r_{i j}(m)$, between $\varepsilon_{i t}^{2}$ and $\varepsilon_{j t-m}^{2}$ for the bivariate UECCC-GARCH $(1,1)$ from Example 2. We set $b_{21}=$ -0.10 and vary $b_{11}$ from -0.70 (lowest, dotted line) to 0 (highest, solid line) by steps of 0.10 .

natural $\log$ of the Consumer Price Index and Industrial Production Index, respectively.

Assuming conditional normality, we estimate an $\operatorname{ARMA}(12,0)$-UECCC$\operatorname{GARCH}(1,1)$ specification. For reasons of brevity, we omit the results for the mean equation. The estimation results for the conditional variances are:

$\mathbf{h}_{t}=\left(\begin{array}{c}0.482 \\ (0.140) \\ 5.028 \\ (3.262)\end{array}\right)+\left(\begin{array}{cc}0.086 & 0.009 \\ (0.018) & (0.002) \\ - & 0.257 \\ & (0.054)\end{array}\right) \boldsymbol{\varepsilon}_{t-1}^{\wedge 2}+\left(\begin{array}{cc}0.888 & -0.010 \\ (0.042) & (0.004) \\ 1.296 & 0.512 \\ (0.491) & (0.065)\end{array}\right) \mathbf{h}_{t-1}$,

with $\rho_{12}=-0.026(0.045)$. The numbers in parentheses are robust standard errors.

The parameter $a_{21}$ was set to zero because it turned out to be insignificant. Interestingly, there is a bidirectional feedback between the two variabilities. In particular, there is strong evidence supporting the Logue and Sweeney (1981) theory that inflation uncertainty has a positive impact on the volatility of growth. In sharp contrast, real variability affects nominal uncertainty negatively, as predicted by, among others, Fuhrer (1997). Clearly, the negative coefficient $b_{12}$ would have been ruled out by the sufficient Bollerslev conditions. It is easy to check that the conditions of Proposition 1 are satisfied for the given parameter combination. 

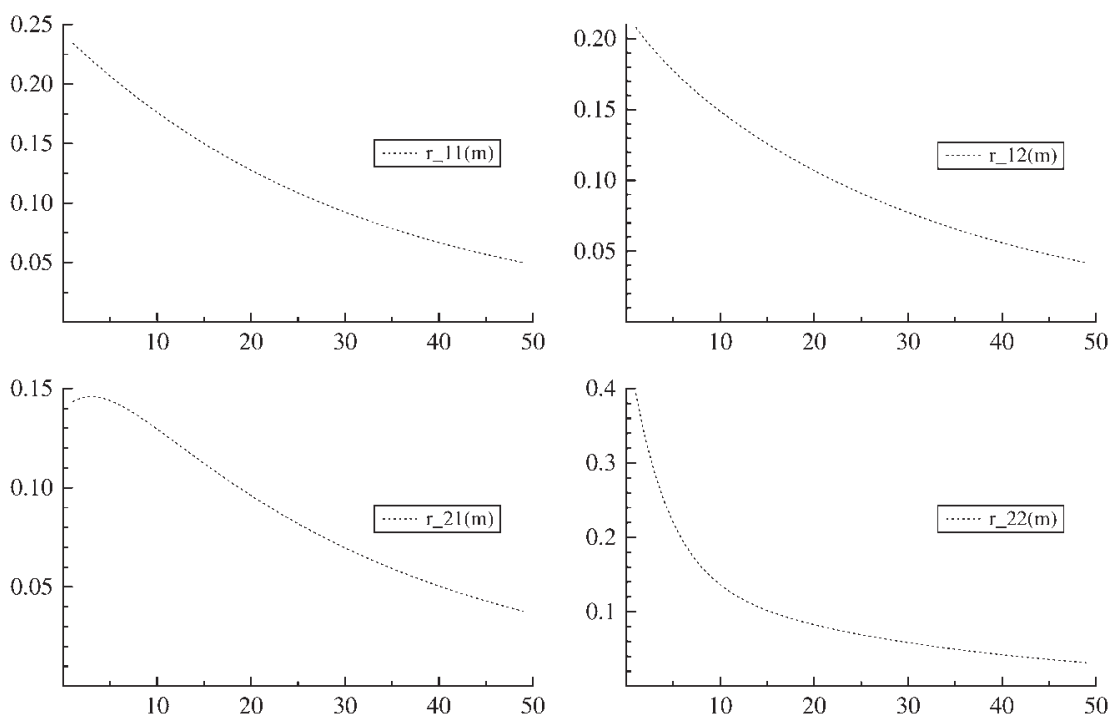

FIGURE 4. The figure shows the $m$ th order auto- and cross-correlations, $r_{i j}(m)$, between $\varepsilon_{i t}^{2}$ and $\varepsilon_{j t-m}^{2}$ for a bivariate process of order $(1,1)$ with parameter estimates as presented in equation (11).

Moreover, the conditions for the existence of the unconditional second and fourth moments are satisfied as well. Finally, it is interesting to note that within the unrestricted extended framework, there exists an intertemporal relation between the two variances whenever the off-diagonal elements of $\mathbf{B}$ are significant. Most importantly, this intertemporal relation implies that the two volatilities are also contemporaneously correlated. This is the case even if the constant conditional correlation coefficient $\rho_{12}$ is insignificant (as in our example). ${ }^{13}$

Figure 4 shows the auto- and cross-correlations of the squared errors. As expected, we observe first an increase in the cross-correlation, $r_{21}(\mathrm{~m})$, between the squared residuals of output and inflation before it starts to decline to zero. This is driven by the strong positive effect from inflation uncertainty to output variability captured by the coefficient $b_{21}$.

\section{CONCLUSIONS}

We have derived necessary and sufficient conditions that ensure the positive definiteness of the conditional covariance matrix in the $N$-dimensional UECCCGARCH model almost surely for all $t$. For this, we have shown that each variance admits an $\operatorname{ARCH}(\infty)$ representation in terms of the $N$ convolutions of the GARCH kernels and the corresponding squared errors. All the $N$ conditional variances are almost surely nonnegative for all $t$ if all the $N^{2}$ kernels are simultaneously nonnegative. It is then straightforward to apply the methodologies 
developed in Nelson and Cao (1992) and Tsai and Chan (2008) to each of these kernels. In contrast to the sufficient Bollerslev conditions, the necessary and sufficient conditions do not rule out the possibility that some of the parameters of the model take negative values. In particular, negative volatility feedback is allowed. We have shown that this substantially increases the permissible parameter space and thereby the flexibility of the model in capturing the stylized facts of economic and financial data. For practical applications, our results suggest that imposing the sufficient Bollerslev conditions on the estimation routine may lead to severe model misspecification. Instead, one should start by estimating the unrestricted model and then check the necessary and sufficient conditions ex post. Alternatively, our conditions can be directly imposed in the maximization procedure. In models of low order, e.g., the bivariate $\operatorname{UECCC-GARCH}(1,1)$, this can be done in a straightforward way. However, as shown in Iglesias and Linton (2007), such constraint maximization procedures create bias terms when one or more of the restrictions are binding, i.e., when the parameter combination lies on the boundary. It would be interesting to investigate whether their bias correction mechanism for univariate GARCH models could be extended to our multivariate framework. ${ }^{14}$

The availability of the necessary and sufficient conditions allows us to test economic theories within the unrestricted framework that were, by construction, excluded in the restricted version. On the other hand, our results also highlight the limitations of the model; e.g., within the bivariate process of order $(1,1)$ it is not possible to test theories that imply negative volatility spillovers in both directions.

We should highlight that our results do not only hold for the CCC process but also for the various parameterizations of the time-varying conditional correlation formulation. Finally, we would like to point to another interesting avenue for future research. Ling and McAleer (2003) derive an asymptotic theory for vector ARMA-GARCH processes under the sufficient Bollerslev conditions. Our results suggest that it would be worthwhile to investigate whether the Ling and McAleer (2003) theory also holds under the necessary and sufficient conditions derived in this paper.

\section{NOTES}

1. Other multivariate GARCH models are the CCC-ARCH process that appears in Cecchetti, Cumby, and Figlewski (1988) or the VECH model of Bollerslev, Engle, and Wooldridge (1988). A multivariate specification that can be viewed as a restricted version of the VECH model is the BEKK formulation defined in Engle and Kroner (1995). Scherrer and Ribaritis (2007) deal with issues of structure and parameterization of VECH and BEKK models.

2. For empirical applications of the extended version, see Nakatani and Teräsvirta (2009).

3. Nonnegativity conditions for the fractionally integrated GARCH (FIGARCH) and the hyperbolic GARCH model can be found in Conrad and Haag (2006) and Conrad (2007), respectively. Finally, nonnegativity conditions for ARMA processes are provided by Tsai and Chan (2007).

4. In a recent article, Gourieroux (2007) also deals with the problem of deriving positivity conditions for multivariate volatility processes. However, his results apply to bivariate autoregressive volatility specifications only. 
5. Similar results were obtained by He and Teräsvirta (1999) and Conrad and Haag (2006) for the univariate GARCH and FIGARCH models, respectively. Both show that the potential shapes of the autocorrelation functions are considerably more flexible under the necessary and sufficient conditions than under the ones that impose nonnegative parameters.

6. We will use the terms variance, variability, uncertainty, and volatility interchangeably in the remainder of the text.

7. Caporin (2007) suggests the exponential causality GARCH model, which allows for negative volatility feedback.

8. For simplicity and without loss of generality, we assume that $b_{i j}^{(p)} \neq 0$ and $a_{i j}^{(q)} \neq 0$ for $i, j=$ $1, \ldots, N$.

9. This version can be obtained from equation (3) as follows: Replace $\mathbf{h}_{t}$ by $\mathbf{h}_{t}^{*}=\left[h_{i t}^{\delta_{i} / 2}\right]_{i=1, \ldots, N}$ and $\varepsilon_{t}^{\wedge 2}$ by $\varepsilon_{t}^{*}=\mathbf{z}_{t}^{*} \odot \mathbf{h}_{t}^{*}$, where $\mathbf{z}_{t}^{*}=\left[\left(z_{i t}-\gamma_{i}\left|z_{i t}\right|\right)^{\delta_{i}}\right]_{i=1, \ldots, N}$ with $\left|\gamma_{i}\right|<1$ and $\delta_{i} \in(0, \infty)$. For the asymmetric power model, Lemma 2 holds with $\mathbf{h}_{t}^{*}=\boldsymbol{\omega} / \beta(1)+\Psi(L) \varepsilon_{t}^{*}$, where the $\operatorname{ARCH}(\infty)$ polynomial $\Psi(L)$ is now multiplied by the nonnegative innovations $\varepsilon_{t}^{*}$ and, hence, Theorem 1 can be directly applied.

10. However, in the trivariate UECCC-GARCH $(1,1)$ model, it is easy to find parameter combinations that satisfy the necessary and sufficient conditions but at the same time imply negative volatility spillovers between two of the three conditional variances.

11. For the time-varying conditional correlation, they employ the equation $\left(1-\gamma_{1} L\right) q_{t}=\gamma_{0}+$ $\gamma_{2} L \varepsilon_{2 t}$ (see also Tsay, 2002, p. 374).

12. The analytic forms for the auto- and cross-correlations of the squared errors of the UECCC$\operatorname{GARCH}(1,1)$ model are given in Corollary 3 in He and Teräsvirta (2004). It is interesting to note that one can express the $N$-dimensional vector $\operatorname{UECCC-GARCH}(p, q)$ model in an ARMA representation and then apply the methodology in Karanasos (1999a, 1999b, 2007) to obtain the correlation structure of the squared errors (see Conrad and Karanasos, 2008).

13. In sharp contrast, an insignificant $\rho_{12}$ in the diagonal CCC model would imply the complete absence of a relation between the variances of inflation and output growth. Studies such as, e.g., Grier and Perry (2000) and Wilson (2006) that employ this formulation tend to find insignificant constant correlations and, hence, miss the link between the two volatilities. Finally, it should be noted that an insignificant constant conditional correlation does not imply the absence of a relationship between the levels of inflation and output growth. Such a link could be at work via cross-effects in the mean equations (economic theories that predict either positive or negative relations are discussed in, e.g., Fountas et al., 2006).

14. Alternatively, if the unrestricted model is estimated and one or several positivity conditions are violated, it may be appropriate to estimate a restricted model by imposing that those inequality constraints that were previously violated hold with equality. If the remaining conditions are satisfied for the restricted model, one can test the restricted against the unrestricted specification. If the restrictions are not rejected, the restricted model can be applied.

\section{REFERENCES}

Bai, J. \& Z. Chen (2008) Testing multivariate distributions in GARCH models. Journal of Econometrics 143, 19-36.

Bauwens, L., S. Laurent, \& J.V.K. Rombouts (2006) Multivariate GARCH models. Journal of Applied Econometrics 21, 79-109.

Bollerlsev, T. (1986) Generalized autoregressive conditional heteroskedasticity. Journal of Econometrics 31, 307-327.

Bollerslev, T. (1990) Modelling the coherence in short-run nominal exchange rates: A multivariate generalised ARCH model. Review of Economics and Statistics 72, 498-505.

Bollerslev, T., R.F. Engle, \& J.M. Wooldridge (1988) A capital asset pricing model with time-varying covariances. Journal of Political Economy 96, 116-131. 
Caporin, M. (2007) Variance (non) causality in multivariate GARCH. Econometric Reviews 26, 1-24.

Cecchetti, S., R. Cumby, \& S. Figlewski (1988) Estimation of the optimal futures hedge. Review of Economic Studies 70, 623-630.

Conrad, C. (2007) Non-Negativity Conditions for the Hyperbolic GARCH Model. KOF Working papers 162, ETH Zurich.

Conrad, C. \& B. Haag (2006) Inequality constraints in the fractionally integrated GARCH model. Journal of Financial Econometrics 4, 413-449.

Conrad, C. \& M. Karanasos (2008) The Correlation Structure of the Squared Errors for the Unrestricted ECCC-GARCH Model. Unpublished paper, Brunel University.

Engle, R.F. (2002) Dynamic conditional correlation: A simple class of multivariate generalised autoregressive conditional heteroscedasticity models. Journal of Business and Economic Statistics 20, 339-350.

Engle, R.F. \& K.F. Kroner (1995) Multivariate simultaneous generalised ARCH. Econometric Theory $11,122-150$.

Fountas, S., M. Karanasos, \& J. Kim (2006) Inflation uncertainty, output growth uncertainty, and macroeconomic performance. Oxford Bulletin of Economics and Statistics 68, 319-343.

Fuhrer, J. (1997) Inflation/output variance trade-offs and optimal monetary policy. Journal of Money, Credit, and Banking 29, 214-234.

Gourieroux, C. (2007) Positivity conditions for a bivariate autoregressive volatility specification. Journal of Financial Econometrics 5, 624-636.

Grier, K. \& M. Perry (2000) The effects of real and nominal uncertainty on inflation and output growth: Some GARCH-M evidence. Journal of Applied Econometrics 15, 45-58.

Hafner, C.M. (2003) Fourth moment structure of multivariate GARCH models. Journal of Financial Econometrics 1, 26-54.

He, C. \& T. Teräsvirta (1999) Properties of the autocorrelation function of squared observations for second-order GARCH processes under two sets of parameter constraints. Journal of Time Series Analysis 20, 23-30.

He, C. \& T. Teräsvirta (2004) An extended constant conditional correlation GARCH model and its fourth-moment structure. Econometric Theory 20, 904-926.

Iglesias, E.M. \& O.B. Linton (2007) Higher order asymptotic theory when a parameter is on a boundary with an application to GARCH models. Econometric Theory 23, 1136-1161.

Jeantheau, T. (1998) Strong consistency of estimators for multivariate ARCH models. Econometric Theory 14, 70-86.

Karanasos, M. (1999a) The Covariance Structure of the S-GARCH and M-GARCH Models. Discussion paper 12, University of York.

Karanasos, M. (1999b) The second moment and the autocovariance function of the squared errors of the GARCH model. Journal of Econometrics 90, 63-76.

Karanasos, M. (2007) The correlation structure of some financial time series models. Quantitative and Qualitative Analysis in Social Sciences 2, 71-87.

Karanasos, M. \& A. Kartsaklas (2009) Dual long-memory, structural breaks and the link between turnover and the extreme-value volatility. Journal of Empirical Finance, forthcoming.

Leboit, O., P. Santa-Clara, \& M. Wolf (2003) Flexible multivariate GARCH modeling with an application to international stock markets. Review of Economics and Statistics 85, 735-747.

Ling, S. \& M. McAleer (2003) Asymptotic theory for a vector ARMA-GARCH model. Econometric Theory 19, 280-310.

Logue, D. \& R. Sweeney (1981) Inflation and real growth: Some empirical results. Journal of Money, Credit, and Banking 13, 497-501.

Nakatani, T. \& T. Teräsvirta (2008) Positivity constraints on the conditional variances in the family of conditional correlation GARCH models. Finance Research Letters 5, 88-95.

Nakatani, T. \& T. Teräsvirta (2009) Testing for volatility interactions in the constant conditional correlation GARCH model. Econometrics Journal 12, 147-163.

Nelson, D.B. \& C.Q. Cao (1992) Inequality constraints in the univariate GARCH model. Journal of Business and Economic Statistics 10, 229-235. 
Scherrer, W. \& E. Ribaitis (2007) On the parametrization of multivariate GARCH models. Econometric Theory 23, 464-484.

Silvennoinen, A. \& T. Teräsvirta (2007) Multivariate GARCH models. In T.G. Anderson, R.A. Davis, J.P. Kreiss, \& T. Mikosch (eds.), Handbook of Financial Time Series. Springer.

Silvennoinen, A. \& T. Teräsvirta (2009) Modelling multivariate autoregressive heteroskedasticity with the double smooth transition conditional correlation GARCH model. Journal of Financial Econometrics 7, 373-411.

Tsai, H. \& K.S. Chan (2007) A note on non-negative ARMA processes. Journal of Time Series Analysis 28, 350-360.

Tsai, H. \& K.S. Chan (2008) A note on inequality constraints in the GARCH model. Econometric Theory 24, 823-828.

Tsay, R.S. (2002) Analysis of Financial Time Series. Wiley.

Tse, Y.K. \& A.K.C. Tsui (2002) A multivariate generalized autoregressive conditional heteroscedasticity model with time-varying correlations. Journal of Business and Economic Statistics 20, 351-362.

Wilson, B.K. (2006) The links between inflation, inflation uncertainty and output growth: new time series evidence from Japan. Journal of Macroeconomics 28, 609-620.

\section{APPENDIX A: Second and Fourth Moments}

A.1. Unconditional Variances. Define $p^{*}=\max (p, q)$, and set $\mathbf{C}_{t}^{(l)}=\left[c_{i j, t}^{(l)}\right]_{i, j=1, \ldots, N}=$ $\mathbf{A}^{(l)} \mathbf{Z}_{t}+\mathbf{B}^{(l)}, l=1, \ldots, p^{*}$, where $\mathbf{Z}_{t}=\operatorname{diag}\left\{\mathbf{z}_{t}^{\wedge 2}\right\}, \mathbf{A}^{(l)}=0$ if $l>q$ and $\mathbf{B}^{(l)}=0$ if $l>p$. Note that $\left\{\mathbf{C}_{t}^{(l)}\right\}$ is a sequence of i.i.d. random matrices (for $\mathbf{A}^{(l)} \neq 0$ ) such that $\mathbf{C}_{t}^{(l)}$ is independent of $\mathbf{h}_{t}$.

By equation (2) we may rewrite equation (3) as

$\mathbf{h}_{t}=\mu+\sum_{l=1}^{p^{*}} \mathbf{C}_{t-l}^{(l)} \mathbf{h}_{t-l}$.

Next we shall make use of the following notation. Let $\Gamma(L)=\mathbf{I}_{N}-\sum_{l=1}^{p^{*}} \Gamma^{(l)} L^{l}$, where $\Gamma^{(l)}=\left[\gamma_{i j}^{(l)}\right]_{i, j=1, \ldots, N}=\mathbb{E}\left(\mathbf{C}_{t}^{(l)}\right)$, with $\gamma_{i j}^{(l)}=a_{i j}^{(l)} \mathbb{E}\left(z_{j t}^{2}\right)+b_{i j}^{(l)} \cdot \operatorname{Set} \gamma(L)=1-\sum_{l=1}^{N \times p^{*}}$ $\gamma_{l} L^{l}=\operatorname{det}[\Gamma(L)]$. We have assumed $\gamma_{N \times p^{*}} \neq 0$, that is, $\gamma(L)$ is a scalar polynomial of order $N \times p^{*}$. Define also $\widetilde{\boldsymbol{\omega}}=\left[\widetilde{\omega}_{i}\right]_{i=1, \ldots, N}=\operatorname{adj}[\Gamma(1)] \mu$.

Assumption A3 (Stationarity). The roots of $\operatorname{det}[\Gamma(z)]$ lie outside the unit circle.

Notice that when $\mathbf{A}^{(r)} \geq 0, r=1, \ldots, q$, the stationarity assumption implies the invertibility condition.

LEMMA 3. When Assumption A3 holds, the unconditional variances of the elements of $\boldsymbol{\varepsilon}_{t}, \boldsymbol{\mu}_{2}=\left[\mu_{2, i}\right]_{i=1, \ldots, N}=\mathbb{E}\left(\mathbf{h}_{t}\right)$, exist if $\widetilde{\boldsymbol{\omega}}>0$, and are given by

$\boldsymbol{\mu}_{2}=\frac{1}{\gamma(1)} \widetilde{\boldsymbol{\omega}}$

Let $\widetilde{\boldsymbol{\omega}}>0$ hold. Then, under Assumption A3, the vector $\operatorname{UECCC-GARCH}(p, q)$ model has a weakly stationary solution. Moreover, this solution is unique and is also strictly stationary and ergodic (see Prop. 3.1 in Jeantheau, 1998). 
Remark 2. Recall that He and Teräsvirta (2004) assume all the parameters in $\boldsymbol{\mu}$ to be positive, and all in $\mathbf{A}(L)$ and $\mathbf{B}(L)$ to be nonnegative. In this situation and under Assumption A3, the positivity of $\widetilde{\boldsymbol{\omega}}$ is guaranteed by construction (see Rmk. 3 in their paper). In sharp contrast, in the unrestricted model we allow some of these parameters to be negative and, hence, the condition $\widetilde{\boldsymbol{\omega}}>0$ has to be checked.

A.2. Fourth-Moment Structure of the Process of Order (1,1). To keep this article relatively self-contained, we briefly review the main theoretical results of $\mathrm{He}$ and Teräsvirta (2004) on the fourth-moment structure of the bivariate UECCC-GARCH $(1,1)$ process defined in equation (5). (The papers by Karanasos, 1990a, and Hafner, 2003, also contain results on fourth moments of multivariate GARCH models.) Equation (A.1) becomes

$\mathbf{h}_{t}=\mu+\mathbf{C}_{t-1} \mathbf{h}_{t-1}$,

where for typographical convenience we have set $\mathbf{C}_{t-1}=\mathbf{C}_{t-1}^{(1)}$.

Assume $\mathbb{E}\left(z_{i t}^{2} z_{j t}^{2}\right)<\infty, i, j=1,2$, and let $\Gamma_{C}=\mathbb{E}\left(\mathbf{C}_{t}\right), \Gamma_{Z \otimes Z}=\mathbb{E}\left(\mathbf{Z}_{t} \otimes \mathbf{Z}_{t}\right)$, and $\Gamma_{C \otimes C}=\mathbb{E}\left(\mathbf{C}_{t} \otimes \mathbf{C}_{t}\right)$. Notice that under the assumption of conditional normality, we have $\Gamma_{C}=\mathbf{A}+\mathbf{B}$ and $\boldsymbol{\Gamma}_{Z \otimes Z}=\operatorname{diag}\left\{3,1+2 \rho_{12}^{2}, 1+2 \rho_{12}^{2}, 3\right\}$.

Moreover, let $\lambda\left(\Gamma_{C \otimes C}\right)$ denote the modulus of the largest eigenvalue of $\Gamma_{C} \otimes C$. Then the matrix $\mathbb{E}\left[\varepsilon_{t}^{\wedge 2}\left(\varepsilon_{t}^{\wedge 2}\right)^{\prime}\right]$ of the fourth moments of $\left\{\boldsymbol{\varepsilon}_{t}\right\}$ exists if

$\lambda\left(\Gamma_{C \otimes C}\right)<1$

and

$\operatorname{adj}\left(\widetilde{\Gamma}_{C \otimes C}\right)\left[\operatorname{vec}\left(\mu \mu^{\prime}\right)+\Gamma_{C}^{\mu}\right]>0$,

where

$\widetilde{\Gamma}_{C \otimes C}=\mathbf{I}_{4}-\Gamma_{C \otimes C}$,

$\Gamma_{C}^{\mu}=\left(\Gamma_{C} \otimes \mu+\mu \otimes \Gamma_{C}\right)\left(\mu^{\prime} \otimes \mathbf{I}_{2}\right) \operatorname{vec}\left(\mathbf{I}_{2}-\Gamma_{C}\right)^{-1}$.

Denote $\boldsymbol{\mu}_{4}=\left[\mu_{4, i j}\right]_{i, j=1,2}=\mathbb{E}\left[\boldsymbol{\varepsilon}_{t}^{\wedge 2}\left(\boldsymbol{\varepsilon}_{t}^{\wedge 2}\right)^{\prime}\right]$. Notice that $\boldsymbol{\mu}_{4}$ is a square matrix. Under equations (A.3)-(A.4),

$\boldsymbol{\mu}_{4}=\Gamma_{Z \otimes Z}\left\{\left(\widetilde{\boldsymbol{\Gamma}}_{C \otimes C}\right)^{-1}\left[\operatorname{vec}\left(\boldsymbol{\mu} \boldsymbol{\mu}^{\prime}\right)+\Gamma_{C}^{\mu}\right]\right\}$.

(see Cor. 2 in He and Teräsvirta, 2004).

Remark 3. Condition (A.4) is needed because by Proposition 1 we allow some of the parameters in the vector $\boldsymbol{\mu}$ and the matrix $\mathbf{B}$ to take negative values. Under the stricter assumption made by He and Teräsvirta (2004), namely, that all parameters of the process are nonnegative, condition (A.4) is directly satisfied.

\section{APPENDIX B: Proofs}

Proof of Lemma 1. Multiply both sides of equation (3) by $\operatorname{adj}[\mathbf{B}(L)]=\beta(L)$ $[\mathbf{B}(L)]^{-1}$.

Proof of Corollary 1. The proof follows immediately from Lemma 1.

Proof of Lemma 2. Simply divide equation (4) by $\beta(L)$. 
Proof of Theorem 1. From the $\mathrm{ARCH}(\infty)$ expansion, that is, equation (7), it follows that each conditional variance, $h_{i t}$, admits an infinite moving-average representation in terms of the $N$ convolutions of the GARCH kernels $\left(\Psi_{i j}(L), j=1, \ldots, N\right)$ and the corresponding squared errors. Thus, the proof follows by applying Theorem 1 in Tsai and Chan (2008) to each of the $N^{2}$ kernels.

Proof of Proposition 1. Initially, we obtain the conditions in terms of the $\omega$ 's, $\alpha$ 's, and $\beta$ 's. That is, $\left(\mathrm{C}^{\prime}\right): \phi_{1} \alpha_{i j}^{(1)}+\alpha_{i j}^{(2)}>0$; $\left(\mathrm{C}^{\prime} \mathrm{a}\right): \psi_{i j}^{(1)}=\alpha_{i j}^{(1)} \geq 0$, and $\left(\mathrm{C}^{\prime} \mathrm{b}\right): \psi_{i j}^{(2)}=$ $\beta_{1} a_{i j}^{(1)}+a_{i j}^{(2)} \geq 0$. Note that since $\beta_{1}=\phi_{1}+\phi_{2}$, if $\phi_{2}>0$, then $\alpha_{i j}^{(1)} \phi_{1}+\alpha_{i j}^{(2)}>0$ directly implies $\psi_{i j}^{(2)} \geq 0$ and vice versa if $\phi_{2}<0$. Finally, using the expressions in equation (6) and the fact that $\phi_{1}=\beta_{1}-\phi_{2}=b_{11}+b_{22}-\phi_{2}$, the conditions are rewritten in terms of the $\mu$ 's, $a$ 's, and $b$ 's.

Proof of Corollary 2. The assumption that $\phi_{1}>\left|\phi_{2}\right|$ implies $\beta_{1}=\phi_{1}+\phi_{2}=b_{11}+$ $b_{22} \geq 0$. Hence, $b_{11}$ and $b_{22}$ cannot be negative simultaneously.

Proof of Corollary 3. Conditions $\left(\mathrm{C}^{\prime}{ }^{\prime} \mathrm{a}\right)$ and $\left(\mathrm{C}^{\prime}\right)$ (when $\left.\phi_{2}>0\right)$ or $\left(\mathrm{C}^{\prime} \mathrm{b}\right.$ ) (when $\left.\phi_{2}<0\right)$ imply that both elements of the same row $\left(b_{i 1}, b_{i 2}, i=1,2\right)$ cannot take negative values.

Proof of Corollary 4. Let the two off-diagonal elements of the $\mathbf{B}$ matrix be negative and the other two positive. We will show that if conditions $\left(\mathrm{C1}^{\prime}\right)$ and $\left(\mathrm{C}^{\prime} \mathrm{a}\right)$ in Proposition 1 are satisfied, conditions $\left(\mathrm{C}^{\prime}\right)$ and $\left(\mathrm{C}^{\prime} \mathrm{b}\right)$ are violated. First we will show that the case $\phi_{2}<0$ or (since $\left.\phi_{1}>0\right) \beta_{2}=-\phi_{1} \phi_{2}=b_{12} b_{21}-b_{11} b_{22}>0$ violates the constraints of condition $\left(\mathrm{C}^{\prime} \mathrm{b}\right)$. Under condition $\left(\mathrm{C}^{\prime} \mathrm{a}\right)$, the latter condition amounts to

$b_{11} a_{11} \geq\left|b_{12}\right| a_{21}, \quad b_{11} a_{12} \geq\left|b_{12}\right| a_{22}$,

$b_{22} a_{21} \geq\left|b_{21}\right| a_{11}, \quad b_{22} a_{22} \geq\left|b_{21}\right| a_{12}$.

Then if we multiply the two inequalities of the first column, we get $b_{11} b_{22} \geq b_{12} b_{21}$, which contradicts $\phi_{2}<0$. Next, we will focus our attention on the case $\phi_{2}>0$. Notice that $\phi_{1}, \phi_{2}>0$ implies that $\beta_{2}<0$ or $b_{12} b_{21}<b_{11} b_{22}$. Since $\phi_{2}>0$, condition (C3'b) is redundant. Under condition ( $\left.\mathrm{C}^{\prime} \mathrm{a}\right)$ and the fact that $\phi_{2}<b_{11}, b_{22}<\phi_{1}$, condition $\left(\mathrm{C}^{\prime}\right)$ amounts to

$$
\begin{array}{ll}
\left(b_{11}-\phi_{2}\right) a_{11}>\left|b_{12}\right| a_{21}, & \left(b_{11}-\phi_{2}\right) a_{12}>\left|b_{12}\right| a_{22}, \\
\left(b_{22}-\phi_{2}\right) a_{21}>\left|b_{21}\right| a_{11}, & \left(b_{22}-\phi_{2}\right) a_{22}>\left|b_{21}\right| a_{12} .
\end{array}
$$

Notice that the two inequalities of the first column imply that $\left|b_{12}\right| /\left(b_{11}-\phi_{2}\right)<a_{11} / a_{21}<$ $\left(b_{22}-\phi_{2}\right) /\left|b_{21}\right|$, while those of the second column imply that $\left|b_{21}\right| /\left(b_{22}-\phi_{2}\right)<a_{22} / a_{12}<$ $\left(b_{11}-\phi_{2}\right) /\left|b_{12}\right|$. Since $\frac{b_{21}}{\left(b_{11}-\phi_{2}\right)}=\left(b_{22}-\phi_{2}\right) / b_{12}$, the four inequalities cannot be satisfied simultaneously.

Proof of Corollary 5. From the previous corollaries, we know that at most two entries in one column of $\mathbf{B}$ may be allowed to be negative. That is because neither the two diagonal, the two off-diagonal, nor the two entries in one row can be negative. Further, assume that conditions $\left(\mathrm{Cl}^{\prime}\right)$ and $\left(\mathrm{C}^{\prime} \mathrm{a}\right)$ hold. Also, without loss of generality, let $b_{11}, b_{21}>0$, and let one or both elements in the second column of the $\mathbf{B}$ matrix be negative. We will examine the two cases where $\phi_{2} \lessgtr 0$. Notice that when only $b_{12}<0$ then $\phi_{2}>0$, whereas when 
only $b_{22}<0$ then $\phi_{2}<0$. Moreover, when $b_{12}, b_{22}<0$ then $\phi_{2} \gtrless 0$ if $\left|b_{12} b_{21}\right| \gtrless\left|b_{11} b_{22}\right|$. If $\phi_{2}<0$, condition $\left(\mathrm{C}^{\prime}\right)$ is redundant and condition $\left(\mathrm{C}^{\prime} \mathrm{b}\right)$ becomes

$a_{11} b_{21} \geq a_{21}\left|b_{22}\right|, \quad a_{12} b_{21} \geq a_{22}\left|b_{22}\right|$.

The above inequalities hold when i) only $b_{22}<0$ and ii) $b_{12}, b_{22}<0$ and $\left|b_{12} b_{21}\right|<$ $\left|b_{11} b_{22}\right|$. Notice that the last inequality implies that $\left|b_{12}\right| / b_{11}<\left|b_{22}\right| / b_{21}$. If $\phi_{2}>0$, we only need to check condition $\left(\mathrm{C}^{\prime}\right)$. This condition reduces to $a_{11} b_{21}>a_{21}\left|b_{22}-\phi_{2}\right|$, $a_{12} b_{21}>a_{22}\left|b_{22}-\phi_{2}\right|$, since $b_{12} /\left(b_{11}-\phi_{2}\right)=\left(b_{22}-\phi_{2}\right) / b_{21}$ and hence $b_{22}<\phi_{2}$. The above inequalities hold when (i) only $b_{12}<0$ and (ii) $b_{12}, b_{22}<0$ and $\left|b_{12} b_{21}\right|>$ $\left|b_{11} b_{22}\right|$.

Proof of Corollary 6. Let condition $\left(\mathrm{C}^{\prime}\right)$ in Proposition 1 hold. Without loss of generality, we will examine the two cases where $b_{11} \lessgtr 1$ (with $b_{22}<1$ ). First, assume $b_{11}<1$. If $b_{12}<0, b_{22} \lessgtr 0$, and the other two parameters in $\mathbf{B}$ are positive, then the two inequalities in equation (9) rule out the possibility that $\mu_{1} \leq 0$. Thus equation (9) becomes

$\mu_{1}>0, \mu_{2} \leq 0, \quad$ and $\quad \frac{\left|\mu_{2}\right|}{\mu_{1}}<\frac{b_{21}}{\left(1-b_{11}\right)}$ or

$\mu_{1}>0, \mu_{2} \geq 0, \quad$ and $\quad \frac{\mu_{2}}{\mu_{1}}<\frac{\left(1-b_{22}\right)}{\left|b_{12}\right|}$.

Note that the inequality in the first expression is more binding than the one in the second expression since, from the invertibility condition, $\left(1-b_{22}\right) /\left|b_{12}\right|>b_{21} /\left(1-b_{11}\right)$. Further, if $b_{22} \lessgtr 0$ and the other three parameters in $\mathbf{B}$ are positive, then the two inequalities in equation (9) rule out the possibility that $\mu_{1}, \mu_{2} \leq 0$. Thus, equation (9) reduces to

$\mu_{1}>0, \mu_{2} \geq 0, \quad$ or $\quad \mu_{1} \geq 0, \mu_{2}>0, \quad$ or

$\mu_{1} \leq 0, \mu_{2}>0, \quad$ and $\quad \frac{\left|\mu_{1}\right|}{\mu_{2}}<\frac{b_{12}}{\left(1-b_{22}\right)}$, or

$\mu_{1}>0, \mu_{2} \leq 0, \quad$ and $\quad \frac{\mu_{2}}{\left|\mu_{1}\right|}<\frac{b_{21}}{\left(1-b_{11}\right)}$,

since from the invertibility condition, $b_{12} /\left(1-b_{22}\right)<\left(1-b_{11}\right) / b_{21}$. Second, let $b_{11}>1$. If $b_{12}<0, b_{22} \lessgtr 0$, and $b_{21}>0$, then the two inequalities in equation (9) rule out the possibility that $\mu_{1} \leq 0$ and $\mu_{2} \geq 0$. In this case, part (a) of Proposition 1 becomes

$\mu_{1} \leq 0, \mu_{2}<0, \quad$ and $\quad \frac{\left|\mu_{1}\right|}{\left|\mu_{2}\right|}<\frac{\left|1-b_{11}\right|}{b_{21}}$ or

$\mu_{1}>0, \mu_{2} \geq 0, \quad$ and $\quad \frac{\mu_{2}}{\mu_{1}}<\frac{\left(1-b_{22}\right)}{\left|b_{12}\right|}$ or

$\mu_{1}>0, \mu_{2} \leq 0$

since from the invertibility condition, $\left(1-b_{22}\right) /\left|b_{12}\right|<b_{21} /\left|1-b_{11}\right|$.

Proof of Lemma 3. Taking expectations on both sides of equation (A.1) yields $\Gamma$ (1) $\mathbb{E}\left(\mathbf{h}_{t}\right)=\mu$. Multiply both sides of this expression by adj $[\Gamma(1)]=\gamma(1)[\Gamma(1)]^{-1}$ to get equation (A.2). 